Instituto Internacional de Investigación y Desarrollo Tecnológico Educativo INDTEC, C.A.

DOI: https://doi.org/10.29394/scientific.issn.2542-2987.2017.0.0.2.29-47

OAI-PMH: http://www.indteca.com/ojs/index.php/Revista Scientific/oai

\title{
Gerencia Educativa Motivacional con Base en la Filosofía del Pensamiento Complejo
}

Autora: Belquis Andreina Toro Hoyos

Universidad Fermín Toro, UFT

belquisatoroh@gmail.com

Barinas, Venezuela

\section{Resumen}

La presente investigación tiene como propósito generar un análisis acerca de la gerencia educativa motivacional con base en la filosofía del pensamiento complejo de Edgar Morín, que propicie fuentes de conocimiento para guiar la gestión escolar hacia procesos de participación y valoración. Metodológicamente es de naturaleza cualitativa con postura analítico descriptiva, de carácter fenomenológico. El tipo de investigación es de campo; las técnicas de recolección son la entrevista a profundidad y el registro de observación, aplicado a tres (3) gerentes educativos, como instrumento la guía de entrevista. Para la interpretación se desarrolló la triangulación de datos. EI análisis se abordó considerando la información y hallazgos encontrados en el discurso gerencial de los informantes claves. Con las interpretaciones realizadas al discurso de los informantes claves, permitió conocer que el personal directivo desconoce competencias inherentes al cargo que ocupa, así como los beneficios que ofrece la filosofía del pensamiento complejo; originando grandes desacuerdos entre los miembros de la institución, afectando la armonía e intercambio de idas que permitan mejorar la calidad educativa. Por ello, se hace necesario ofrecer un análisis que permita la reflexión y orientación del rol gerencial hacia el logro de la efectividad, además fomentar un clima adecuado de trabajo. Palabras clave: gerencia educativa; motivación; pensamiento
complejo. Palabras clave: gerencia educativa; motivación; pensamiento
complejo. 


\title{
Motivational Educational Management Based on the Philosophy of Complex Thinking
}

\begin{abstract}
The present research aims to generate an analysis about motivational educational management based on Edgar Morin 's complex thinking philosophy, which provides sources of knowledge to guide school management towards participation and assessment processes. Methodologically, it is qualitative in nature, with descriptive analytical posture, of a phenomenological nature. The type of research is field; the collection techniques are the in-depth interview and observation log, applied to three (3) educational managers, as the interview guide instrument. Triangulation of data was developed for interpretation. The analysis was approached considering the information and findings found in the managerial discourse of the key informants. With the interpretations made to the key informants' discourse, it was possible to know that managers do not know the competences inherent to the position they occupy, as well as the benefits offered by the philosophy of complex thinking; Causing great disagreements between the members of the institution, affecting the harmony and exchange of trips that allow to improve the educational quality. Therefore, it is necessary to offer an analysis that allows the reflection and orientation of the managerial role towards the achievement of effectiveness, in addition to promoting an adequate working climate.
\end{abstract}

Keywords: educational management; motivation; complex thinking.

Date Received: 25-11-2016

Date Acceptance: 06-01-2017 


\section{Introducción}

Vivimos en un mundo que constantemente está cambiando; a través de la historia, el ser humano ha tratado de comprender, interpretar y aprovechar esos cambios para vivir mejor, mediante un conocimiento del mundo que le rodea y dominio más eficaz del mismo. Sacristán (2009), afirma que "las nuevas exigencias y condiciones de la sociedad basada en la información remueven drásticamente los fundamentos de la escuela clásica y de sus modos de entender el conocimiento" (pág. 60). En este sentido la educación juega un papel estelar, puesto que debemos conocer qué, cómo y por qué se educa en una sociedad global, reconociendo las potencialidades de cada uno de los seres humanos.

Actualmente las instituciones educativas venezolanas, cada vez son de mayor competitividad, un personal altamente motivado resulta necesario e indispensable para toda institución que desea obtener resultados satisfactorios, de tal manera que deben esmerarse en estimular un ambiente positivo de trabajo. Por otra parte, es preciso mencionar que la motivación o desmotivación en las personas está relacionada con procesos mentales de cada individuo que determinan sus formas de responder antes estímulos externos, dicha respuesta es diferente en cada ser humano por su propia historia y circunstancia actual, sin embargo existen condicionantes externos que pueden ser controlados por los gerentes educativos y que día a día emiten mensajes propicios para la motivación o desmotivación de las personas que lo integran; por esta razón, es necesario que el gerente ponga de manifiesto las mejores herramientas discursivas como una manera de fortalecer el intercambio comunicacional y a su vez el comportamiento laboral dentro de la institución y por consiguientes optimizar la calidad educativa; la cual va a depender de una buena administración y gerencia.

En consecuencia, los líderes entre las competencias que deben desarrollar, figuran el de aprender a motivar a sus trabajadores. De esta 
manera, con relación a las capacidades que debe poseer los gerentes educativos Páez (2007), expone que:

Dentro de las competencias intelectuales más importantes que tiene un líder en las funciones como tal, está la capacidad de concebir y adoptar decisiones con un alto grado de asertividad... entendiéndose una decisión como la selección de la mejor opción entre todas las disponibles para garantizar la consecución de los objetivos estratégicos formulados dentro de la organización (pág. 11).

De lo antes señalado se infiere que, quienes están inmersos en el mundo organizacional, saben que el factor clave para el éxito o fracaso de una gestión se ubica en la gente (entendida como recurso humano), predecir cómo se desempeñarán y cómo poder controlar su comportamiento sería la manera más efectiva de cumplir con los propósitos de la organización. Para ello, se considera, que existe la necesidad de un nuevo modo de pensar, incluyendo al paradigma de la complejidad, el cual no se rige solo por el pensamiento y los usos lógicos, es decir, el conocimiento, sino también nuestras acciones en la praxis.

Ahora bien, mediante la observación directa se pudo predecir que el origen de esta problemática radica en que el personal que cumple funciones directivas demuestran no estar aptos para los cambios que demandan el entorno, ni para afrontar situaciones de complejidad que requieren una rápida y oportuna respuesta; puesto que se ha observado que la gerencia dentro de las instituciones de educación primaria, pertenecientes al municipio Bolívar del estado Barinas; hasta ahora se carece de factores motivacionales que induzcan al logro del trabajo organizado, colectivo y por ende efectivo, es decir, mantienen una comunicación exigua con el personal a menos que sea por razones de solicitud de recaudos administrativos, convocatorias e informaciones emanadas de la supervisión, acarreando como consecuencia 
que el personal no se sienta tomado en cuenta y por consiguiente, motivado en sus funciones; careciéndose de una relación de calidad.

De acuerdo a lo descrito anteriormente, se genera la necesidad que los directivos educativos propicien incentivos afectivos, morales y éticos que les permitan a los docentes adoptar una actitud positiva ante el trabajo pedagógico, el cual, requiere de personas capaces de sobresalir en momentos de crisis, superar los conflictos, orientando el accionar educativo desde su parte didáctica -pedagógica. Desde luego, ello se atribuye en gran medida a la puesta en práctica de los conocimientos gerenciales por parte del personal directivo de las instituciones de educación primaria, en cuanto al manejo y orientación de su personal desde la perspectiva del pensamiento complejo.

Es por ello, que se propone la práctica de la gerencia educativa motivacional con base en la filosofía del pensamiento complejo, que conlleve al desarrollo de una gestión de calidad; con el fin de lograr un cambio de actitud, y al mismo tiempo la búsqueda de alternativas para contrarrestar el desgano, la apatía y desinformación que hasta ahora se viene sosteniendo.

En virtud a lo antes planteado, y una vez señalada la problemática de estudio se plantean los siguientes enunciados holopráxicos:

¿Cuál es el estado actual de conocimientos que poseen los directivos de educación primaria en cuanto a la gerencia educativa motivacional con base en la filosofía del pensamiento complejo?

¿Cuáles son los fundamentos teóricos de la gerencia educativa motivacional con base en la filosofía del pensamiento complejo?

¿Cómo será el análisis acerca de la gerencia educativa motivacional con base en la filosofía del pensamiento complejo? 


\subsection{Objetivo de la Investigación}

\section{Propósito Rector}

Generar un análisis acerca de la gerencia educativa motivacional con base en la filosofía del pensamiento complejo.

\section{Gerencia Educativa Motivacional}

Actualmente la educación venezolana frente a los retos o desafíos planteados, exige una formación gerencial transdisciplinaria, donde el gerente o director educativo tenga una visión compartida hacia el manejo de una serie de contenidos que le permite el abordaje efectivo de la gestión escolar.

En este sentido, uno de los grandes retos que tiene que enfrentar el personal directivo es de cambios de paradigmas, lo que requiere de la creatividad e innovación en la forma de dirigir y planificar la acción de las relaciones interpersonales, de tal modo que propicie un ambiente de satisfacción y cordialidad en las organizaciones educativas. Estos aspectos exigen una acertada visión curricular, que permita realizar una práctica activa reflexiva y flexible, con un método acorde con las necesidades del personal a su cargo, esas acciones deben contribuir a la modificación de los ambientes socioeducativos, mediante el ejercicio de un liderazgo efectivo, que permitirá propiciar un clima motivacional optimo en términos de eficacia y eficiencia para la obtención de resultados satisfactorios en los procesos educativos.

Es importante acotar, que actualmente en el ámbito de la educación venezolana en la praxis de la gerencia educativa, aún persiste una acción gerencial controladora, rígida, vertical, tímida y eminentemente administrativa, que va en detrimento de lo pedagógico, esto contribuye al deterioro de la calidad educativa. De allí, que se plantea la necesidad de renovar la acción gerencial a través del espíteme de la gerencia educativa desde el enfoque de la complejidad, a fin de que el gerente educativo se convierta en un eje 
dinamizador para la transformación de la práctica educativa mediante la reflexión, la motivación y el liderazgo que inspire a las maestras y maestros asumir la educación como compromiso social.

Desde esta perspectiva el líder educativo debe inspirar confianza, solidaridad, motivación y buenas relaciones interpersonales, para que su liderazgo como docente directivo sea optimo y contribuya a los objetivos propuestos de su institución. Con referencia a lo anterior Chiavenato (2001) plantea que la:

"Gerencia Educativa: puede concebirse, como el proceso a través del cual se orienta y conduce la labor docente y administrativa de la escuela, y sus relaciones con el entorno, con miras a conseguir los objetivos institucionales mediante el trabajo de todos los miembros de la comunidad, a fin de ofrecer un servicio de calidad, y coordinar las distintas tareas y funciones de los miembros hacia la consecución de proyectos comunes." (pág. 18).

En tal sentido, para el logro de un proceso educativo de calidad se conjugan una serie de aspectos que transcienden más allá de las aulas de clase, donde la efectividad de las instituciones educativas radica en tener una misión y enfoque académico claro, contar con directores que sean lideres formativos, proactivos al cambio, y estimuladores de todo el personal para que participe en la ejecución de éste; adaptarse a los avances y transformaciones económicas, científicas, tecnológicas, sociales, políticas y culturales de la sociedad actual, con el propósito de brindar una educación óptima.

En tal sentido, Alvarado (2009), expresa que:

"Los directivos de las instituciones deben ser funcionarios técnica y humanamente capacitados, creativos e innovadores y con una actitud permanente hacia el cambio, con excelente motivación que exprese su compromiso e iniciativa hacia la tarea educativa, con habilidades sociales o capacidades que 
induzcan respuestas positivas a los demás y se exprese en la influencia que logra" (pág. 13).

Si se cumple con esta premisa, así como con otras que combinen conocimientos y habilidades naturales del directivo, puede deducirse que se obtendría la optimización del desempeño de los directores educativos en beneficio de la organización escolar y de la comunidad circundante. Es de esta forma como se configura el perfil más consensuado del deber ser del gerente escolar.

Por lo tanto, el modelo deseable para los gerentes educativos del tercer milenio es aquel que aplique el liderazgo transformacional, en el cual según Rivero (1998), "la motivación surge por la expresividad del líder, se estimula la creatividad y el compromiso intelectual social, se promueve la actuación de otros, se celebra logros y se reconocen las contribuciones individuales" (pág. 108). Estas son las características del futuro docente si se desea que sea agente poderoso y responsables de los cambios que vienen. Ser líder transformacional es la única vía para convertirse en un guía autentico del proceso educativo.

En este sentido Moss citado por Sánchez (2000), plantea que "es necesario dejar de pensar en gente y se comience a pensar en talento, en nutrir sus metas y sus almas" (pág. 46). Plantea que se requieren cinco (5) poderes para enfrentarse exitosamente a una sociedad cambiante. Estos cinco poderes, que personalmente ha integrado en un concepto que denominó "La Mano del Poder Humano", son:

El poder de la voz, dentro del cual Moss citado por Sánchez, (2000) afirma que "más que organizaciones de aprendizaje, lo que se necesita es organizaciones de educación, donde la voz de quienes más saben o están preparados se traduzca en el vehículo para facultar cada vez más personas en las competencias claves del negocio" (pág. 49) 
El poder de la imaginación; en el cual comenta que aquellas personas que tengan la capacidad de vislumbrar un presente y futuro diferente, más rápido, más efectivo, más rentable y más placentero, serán las que deberán regir las acciones de liderazgo en el nuevo milenio.

El poder de la retribución; donde Moss citado por Sánchez, (2000) expresa que:

"Cada vez, más empresas están realizando acciones sociales de retribución a las comunidades con las cuales interactúan, la razón es que el personal desarrolla un sentido de conexión con la gente que eleva el espíritu y la motivación, aumentando la identificación con la organización y la productividad global" (pág. 51).

Así, como lo planteado por éste autor, las personas que hacen vida en cualquier organización necesitan estímulos que promuevan la participación activa dentro de la misma y, de esta manera alcanzar los mejores resultados hacia el logro de los objetivos propuestos.

El poder del compromiso; si queremos desarrollar el sentido de compromiso en nuestro personal se requiere poner en práctica ciertas condiciones que despierten el poder del talento humano: la propiedad del trabajo, la identidad para con la organización y sus proyectos, la autonomía de acción, la libertad de compartir ideas y hacer cambios, además la posibilidad de acceder a que empleado se sienta dueño y parte del proceso.

El poder de la asociación; implica el hecho de establecer, desarrollar y conservar alianzas, que permitan alcanzar de manera más efectiva y productiva os objetivos prepuestos. 


\subsection{Las funciones del gerente}

En cuanto a lo referido de las funciones de gerente, Fermín y Rubino (1997), enfatizan tres grupos de funciones que son fundamentales para el logro de una efectiva acción gerencial:

1. La creación de un grupo de trabajo armónico donde el todo sea más que la suma de sus partes. Una entidad productiva que rinda más que la suma de los recursos incorporados a la misma.

2. Ser proactivo, es decir, armonizar en todas las decisiones y todos los actos los requerimientos del futuro inmediato y a largo plazo.

3. Ejecutar seis tareas básicas: fijar objetivos; derivar metas en cada área de objetivos; organizar tareas, actividades y personas; motivar y comunicar, controlar y evaluar; y, desarrollar a la gente y a sí mismo.

Por tal motivo, la efectividad de una organización depende claramente de la eficacia y la eficiencia con que el gerente desarrolle sus acciones, al igual de su capacidad para conocer el desempeño laboral y dirigir el comportamiento de las personas que conforman su colectivo de trabajo, generalmente con aptitudes, actitudes y necesidades diferentes, por la vía que conduzca hacia la efectividad de la organización.

De acuerdo a lo mencionado anteriormente, podemos decir que un gerente efectivo es aquel que comunica con sus colaboradores o trabajadores los objetivos y prioridades de la organización, utiliza el consenso para establecer acuerdos comunes, promueve la participación activa de sus colaboradores en la planificación, toma de decisiones y solución de problemas, procura mejorar continuamente la comunicación, que le permita alcanzar un nivel de compromiso e identidad con la organización, analiza y evalúa, conjuntamente con los autores y actores, los logros alcanzados, las causas de las desviaciones y las posibles medidas para mejorar, enlaza logros con recompensas de una manera justa y objetiva, ejerce el control sin perder su 
función de orientador para que puedan realizar eficientemente sus tareas, delega tanto las funciones como el poder para tomar decisiones, brindando autonomía de acción a su grupo de trabajo, cuando se presentan conflictos los afronta aplicando principios empíricos, lógicos y racionales para resolverlos; Considerando los errores tanto propios como los ajenos, como una oportunidad para aprender y mejorar que fortalezca la organización.

\subsection{Filosofía del pensamiento complejo}

La complejidad siempre está presente en toda organización, puesto que, la ciencia de la complejidad tiene mucho que ver con las conexiones y las múltiples interacciones entre las diferentes disciplinas científicas y el desarrollo organizacional de la gerencia en la denominada postmodernidad. La complejidad como enfoque epistemológico encuentra su mayor exponente en el filósofo francés Edgar Morín, cuyo planteamiento puede resumirse según Feliú, (2003) como la búsqueda de:

“... la comprensión del mundo fenoménico a través de una integración de sus eventos pues concibe la realidad como un tejido de constituyentes heterogéneos inseparablemente asociados donde encontramos presente la paradoja de lo uno y lo múltiple. La complejidad intenta abordar las relaciones entre lo empírico, lo lógico y lo racional" (pág. 1).

En este sentido, se plantea la necesidad de renovar la acción gerencial a través del enfoque de la complejidad, a fin de que el gerente educativo o director educativo se convierta en un eje dinamizador para la transformación de la práctica educativa mediante la reflexión, la motivación y el liderazgo que inspire a las maestras y maestros asumir la educación como compromiso social.

Es evidente entonces, que la complejidad se presenta con los rasgos inquietantes de lo enredado, de lo inextricable, del desorden, la ambigüedad la incertidumbre. De allí, la necesidad para el conocimiento, de poner orden 
los fenómenos rechazando el desorden, de descartar lo incierto, de seleccionar los elementos de orden y de certidumbre, de quitar la ambigüedad, clarificar, distinguir, jerarquizar, en fin, lo que se pretende con la complejidad organizacional es afrontar lo entramado para sustituir al paradigma de disyunción, reducción, unidimensionalización por un paradigma de conjunción, distinción que permita distinguir sin desarticular, asociar sin identificar 0 reducir.

De esta manera, se puede expresar que en la concepción de la complejidad organizacional de la nueva gerencia, el gerente juega un gran papel y función importante porque está condicionada a la interpretación y al manejo inteligente de los referentes gerenciales que se presenten en la dinámica social y el desarrollo organizacional, donde la complejidad se evidencia mayormente como dije antes, en el gerente por ser el foco de la realidad que demanda mayor participación, reconocimiento, apertura, especialización; dedicación, compromiso, clara comunicación, respeto por la dignidad humana, y otros valores que puedan ser incluidos en el proceso del cambio gerencial.

\section{Contexto Metodológico}

La perspectiva metodológica que se ha seguido en este estudio ha sido la cualitativacon postura paradigmática interpretativa, la cual según Sandin (2003) es aquella "orientada a la comprensión en profundidad de fenómenos educativos y sociales, a la transformación de prácticas y escenarios socioeducativos, a la toma de decisiones y también hacia el descubrimiento y desarrollo de un cuerpo organizado de conocimientos" (pág. 123). En esta perspectiva se pretende comprender la experiencia propia de cada gerente y los factores que inciden en el fenómeno educativo. 


\subsection{Referente Metodológico}

La investigación de acuerdo a la necesidad planteada, las cuales surgen de las interrogantes emergentes, se ubica en el tipo de investigación de campo, con carácter descriptivo; ajustándose a lo que señala Rangel (2001) "Comprende la descripción, registro, análisis e interpretación de la naturaleza actual, composición o procesos de los fenómenos" (pág. 43). Por tal razón, se puede decir que, es de campo de acuerdo los datos son obtenidos directamente de la situación en estudio.

Con base en las consideraciones anteriores y apoyando que la experiencia vivida es esencialmente un proceso interpretativo, se evoca a la hermenéutica como el arte de interpretar y comprender una situación o realidad en cualquiera de sus manifestaciones; convirtiéndose en el instrumento principal para la obtención y el análisis de los datos, que se deben estudiar con profundidad interpretativa y analíticamente fenomenológica, la cual trata de entender la realidad social como la perciben las personas.

\subsection{Contexto e informantes claves de la Investigación}

El contexto se ubica en el sistema educativo venezolano, concretamente en el nivel Educación Primaria, en las instituciones pertenecientes a la Parroquia Barinitas, Municipio Bolívar del Estado Barinas.

Por el tipo de estudio a realizar se considera que la población son todos los sujetos involucrados en la investigación y guardan relación con el estudio que se realiza, permitiendo el desarrollo de ella y la selección de informantes claves como muestra. En este sentido, según Hernández, Fernández y Baptista (2008), la muestra es un "subgrupo de la población en el que todos los elementos de ésta tienen la misma posibilidad de ser elegidos" (pág. 189). Para el estudio se empleó un muestreo intencionado, puesto que el investigador seleccionó a una parte de los sujetos de acuerdo a las necesidades del estudio. Donde los sujetos informantes fueron tres (3) 
directivos, inmersos en el quehacer como gerentes educativos. Cada uno de ellos con características significativas para ser convertidos en informantes claves.

\subsection{Obtención y Registro de la Información.}

Con el propósito de recabar los datos e información de manera precisa, en la investigación la técnica se basará en primera instancia en la observación directa y seguidamente se hace necesario la aplicación de la entrevista a profundidad la cual es definida por Martínez (2000) como "un instrumento técnico, de gran sintonía epistemológica con este enfoque, y también con sus teorías metodológicas adaptada a un diálogo coloquial, flexible, dinámico, libre y directo" (pág. 65).

\subsection{Validez y Credibilidad de los Instrumentos}

En la investigación se tomó la validez de criterio o denominada juicio de expertos que consiste en entregarle a éstos el instrumento. En este sentido, se les solicitó a los doctores su apoyo para la valoración del instrumento. Con ello los expertos establecieron que había pertinencia y coherencia entre las unidades de análisis, las categorías y las subcategorías, señalando que se adaptaban a los preceptos teóricos que involucran las mismas categorías.

Por su parte, la credibilidad del instrumento se refiere al grado en que su aplicación repetida del instrumento al mismo sujeto produce resultados iguales. Es por ello, que este se consideró pertinente aplicar a tres (3) docentes en función directiva.

\subsection{Análisis, Reflexión e Interpretación de la Realidad}

Una vez aplicados los instrumentos y obtenidos los datos e informaciones sobre el objeto de estudio, se procedió a realizar la descripción de los fenómenos, el estudio analítico de contenidos y la activación de 
procesos reflexivos. Para ello se aplicó el procedimiento desplegado en cuatro etapas que permitan la emergencia de análisis. Estas etapas consisten en, categorizar las unidades de análisis, estructurar, contrastar y el análisis propiamente dicho.

\section{Consideraciones Finales}

Una vez realizado el análisis, comprensión e interpretación del fenómeno objeto de estudio se ha podido constatar que el personal que cumple funciones directiva desconoce competencias inherentes al cargo que ocupa, así como, los beneficios organizativos que brindaría emplear la filosofía del pensamiento complejo en la gerencia educativa, puesto que, en su mayoría éstos no han asumido dichos cargos de acuerdo a lo establecido en la normativa legal, y por consiguiente, no poseen la formación académica ni la experiencia profesional que los conlleve a optimizar sus funciones para alcanzar el éxito de la organización educativa. En lo concerniente a las cualidades humanas, se pudo observar que las relaciones interpersonales en cuanto a la unión entre el personal no son del todo satisfactorias. En consecuencia, origina apatía y desacuerdos entre los miembros de la institución, afectando la armonía y tranquilidad de la organización. Así como, el intercambio de idas necesarias que permitan mejorar la calidad educativa.

Ante la situación planteada, se destaca la necesidad de una gerencia con capacidad productiva, acordes a las actuales demandas educativas, donde los docentes que cumplen funciones directivas deben estar atentos y abiertos a cambios día a día, y no persistir en seguir modelos y patrones que solo sirven para enmarcar la educación, aprovechando las nuevas teorías para complementar las anteriores y así lograr la socio construcción de saberes, donde el gerente de educación primaria mantenga una comunicación y relación personal efectiva con todos los miembros de la institución, a fin de motivar y proyectar a su personal confianza y compromiso con la labor 
organizativa, y permita mejorar el ambiente organizacional de la institución educativa.

De tal manera, que desde lo social, y lo educativo; estos elementos analizados determinaron que la aplicación y manejo de habilidades motivacionales, considerando cada uno de los elementos que brinda la filosofía del pensamiento complejo en la indagación de posibles soluciones para superar esta problemática es viable, dado que busca comprender la esencia de la situación, para luego generar herramientas que permitan obtener la integración, el compromiso y participación activa que van en función del desarrollo de la institución educativa.

En efecto, dentro el ámbito educativo se hace necesario este tipo de estudios debido a que pretende nutrir la formación de los directivos, a fin de contribuir con las herramientas que se deben considerar durante su praxis, para mejorar el desempeño de los mismos, lo cual redundara en beneficios educativos. De esta manera ayudará a desarrollar una gestión compartida, que permita ejercer y aplicar el principio de cooperación, compromiso, solidaridad, entre otros valores en los profesionales de la docencia pertenecientes a la institución y, que a su vez generará una mejor calidad educativa.

En este sentido, y con relación a lo antes planteado, se recomienda que los gerentes educativos deben cumplir con los requisitos establecidos para ocupar dichos cargos, en consecuencia, puedan estar aptos y capacitados para llevar a cabo con efectividad y eficiencia las funciones correspondientes. De tal manera, que los fortalezca institucionalmente, manteniéndose así siempre motivados y facultados para afrontar con éxito el trabajo educativo. Asimismo, se hace necesario que los gerentes conozcan a su personal e interactúen con ellos, a fin de lograr habilidades para crecer y mantener buenas relaciones interpersonales que aportan beneficio tanto a la institución como al proceso educativo, creando mecanismos motivacionales que permitan 
mejorar el desempeño laboral en los docentes, orientados hacia el logro de las metas propuestas por la institución.

En conclusión considero que los trabajos con este tipo de investigación deben darse a conocer y considerarse en las instituciones educativas objeto de estudio, por la relevancia que engloba como alternativas de solución para las dificultades que se presentan día a día dentro de la gerencia educativa, que a su vez, puedan convertirse en un punto de partida para reflexionar, mejorar el desempeño y por consiguiente la motivación dentro de toda organización educativa que pretenda establecer claramente las metas, mediante discusiones dirigidas a concretar ideas y tomar decisiones acertadas; a fin de contribuir con la formación de los líderes del sistema educativo, cuyos perfiles deben estar en consonancia con los cambios y reformas educativas que el país requiere para su desarrollo. De tal manera, que el desempeño del directivo, docente e institución en general sea eficaz y eficiente para alcanzar una escuela exitosa y por tanto brindar una educación de calidad.

\section{Referencias}

Alvarado, L. (2009). Modelo de gerencia corporativa para el equipo itinerante de integración. Trabajo de investigación. Universidad Bicentenaria de Aragua.

Chiavenato, I. (2001). Administración de Recursos Humanos. Editorial McGraw-Hill. México.

Feliú, M. (2003). Entrevista con Edgar Morín: El Desafío de la Complejidad. En el marco del encuentro internacional "Movilizando el Capital Social y el Voluntariado". Recuperado de:

http://webcache.googleusercontent.com/search?q=cache:http://www.iu tep.tec.ve/uptp/images/Descargas/materialwr/articulos/EdgarMorin-

Entrevista.pdf\&gws $\quad r d=c r \& e i=A K S O W M S s H 46 g j w P W-K h Y$ 
Fermín, I., y Rubino, N. (1997). Gerencia y Liderazgo. Material Instruccional. Caracas: UPEL-IPC.

Hernández R., Fernández C., y Batista P. (2008). Metodología de la investigación. (4ta Edición). México: McGraw-Hill.

Martínez, M. (2000). El Paradigma Emergente. D/F. México: Editorial Trillas. Páez, J. (2007). Complejidad Organizacional de la Nueva Gerencia. Gerencia en Acción. Editado por El Carabobeño. Valencia.

Rangel, H. (2001). Intervención y Metodologías. Editorial Manantial. Caracas, Venezuela.

Rivero, L. (1998). Administración, Una Perspectiva Global. 11va Edición. México.

Sacristán, J. (2009). Educar por Competencias. Segunda Edición. Ediciones Morata, S. L. Madrid, España.

Sánchez, F. (2000). Desarrollo Personal. Revista Calidad Empresarial, No. 18, Caracas, Venezuela: Corporación Calidad 2010, C.A.

Sandin, M. (2003). Investigación Cualitativa en Educación. España: Editorial McGraw-Hill. 


\section{Belquis Andreina Toro Hoyos}

e-mail: belquisatoroh@gmail.com

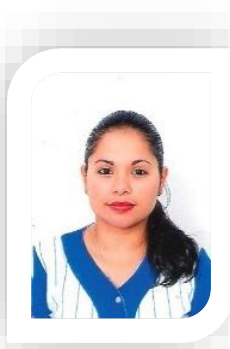

Natural de Barinitas, estado Barinas, Venezuela. Licenciada en Educación mención Geografía e Historia egresada de la Universidad Experimental de los Llanos Occidentales Ezequiel Zamora (Unellez - Barinas), Magister Scientiarum en Gerencia y Liderazgo en Educación por la Universidad Fermín Toro y actualmente doctorando en Ciencias de la Educación por esa misma casa de estudio. Desempeñándome como docente de aula en la Unidad Educativa "José Ramón Traspuesto" en Barinitas estado Barinas, participante en diferentes cursos y jornadas de formación profesional como introducción a la filosofía, elaboración de software educativo, edumática, introducción al sistema operativo Linux, globalización de la educación, socialización del conocimiento en el área de ciencias sociales, desafíos y retos en la gerencia educativa del siglo XXI, entre otros.

El contenido de este manuscrito se difunde bajo una Licencia de Creative Commons ReconocimientoNoComercial-Compartirlgual 4.0 Internacional 